\title{
Tailored circulatory intervention in adults with pulmonary hypertension due to congenital heart disease
}

\author{
L. E. Couperus · I. R. Henkens • M. R. M. Jongbloed • M. G. Hazekamp • M. J. Schalij • H. W. Vliegen
}

Published online: 20 April 2016

(c) The Author(s) 2016. This article is available at SpringerLink with Open Access

\begin{abstract}
Background Adults with pulmonary hypertension associated with congenital heart disease (PH-CHD) often have residual shunts. Invasive interventions aim to optimise pulmonary flow and prevent right ventricular failure. However, eligibility for procedures strongly depends on the adaptation potential of the pulmonary vasculature and right ventricle to resultant circulatory changes. Current guidelines are not sufficiently applicable to individual patients, who exhibit great diversity and complexity in cardiac anomalies.

Methods and Results We present four complex adult PHCHD patients with impaired pulmonary flow, including detailed graphics of the cardiopulmonary circulation. All these patients had an ambiguous indication for shunt intervention. Our local multidisciplinary Grown-Ups with Congenital Heart Disease team reached consensus regarding a patient-tailored invasive treatment strategy, adjacent to relevant guidelines. Interventions improved pulmonary haemodynamics and short-term clinical functioning in all cases.

Conclusions Individual evaluation of disease characteristics is mandatory for tailored interventional treatment in $\mathrm{PH}$ CHD patients, adjacent to relevant guidelines. Both strict
\end{abstract}

L. E. Couperus $(\bowtie) \cdot$ I. R. Henkens · M. R. M. Jongbloed · M. J. Schalij · H. W. Vliegen

Department of Cardiology, Leiden University Medical Center Leiden, Netherlands

e-mail: 1.e.couperus@lumc.nl

M. R. M. Jongbloed

Department of Anatomy, Leiden University Medical Center

Leiden, Netherlands

M. G. Hazekamp

Department of Cardiothoracic Surgery, Leiden University

Medical Center

Leiden, Netherlands registration of cases and multidisciplinary and multicentre collaboration are essential in the quest for optimal therapy in this patient population.

Keywords Pulmonary hypertension - Congenital heart defects · Right ventricular dysfunction · Intervention

\section{Background}

Improvements in medical treatment have changed outcomes in congenital heart disease (CHD), with increasingly complex CHD patients reaching adulthood [1-3]. This rise is accompanied by an increase in the prevalence of CHDrelated comorbidities such as pulmonary hypertension [3, 4]. Pulmonary hypertension especially develops in CHD patients with a persistent shunt between the systemic and pulmonary circulation and is caused by right ventricular (RV) volume and pressure overload, eventually resulting in pulmonary vascular remodelling and increased pulmonary vascular resistance (PVR) [5-7]. The aim of intervention in patients with pulmonary hypertension associated with congenital heart disease (PH-CHD) is to optimise pulmonary flow and prevent RV failure. Targeted therapy for pulmonary hypertension consists of prostacyclins, endothelin receptor antagonists and phosphodiesterase-5 inhibitors and can improve functional status in $\mathrm{PH}-\mathrm{CHD}$ patients [8-10]. Paradoxically, it is suggested that targeted therapy and the consequential reduction in PVR can cause an increase in pulmonary shunt flow and may result in progression of pulmonary endothelial damage $[5,11]$. An alternative approach is to alter shunt size through invasive correction of the circulation, as for instance through closure of a ventricular septum defect (VSD). The aim of such a procedure is to decrease $\mathrm{RV}$ pressure and optimise true pulmonary flow. 


\section{Advertisement placed here.}

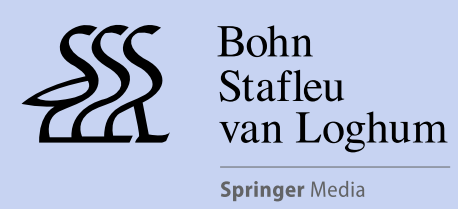

Houten 2016 


\section{Advertisement placed here.}

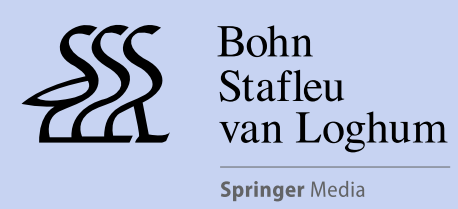

Houten 2016 


\section{Advertisement placed here.}

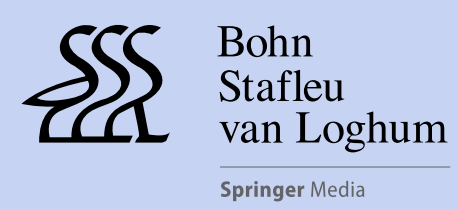

Houten 2016 


\section{Advertisement placed here.}

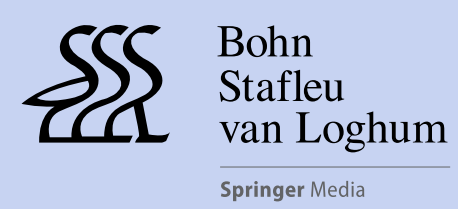

Houten 2016 

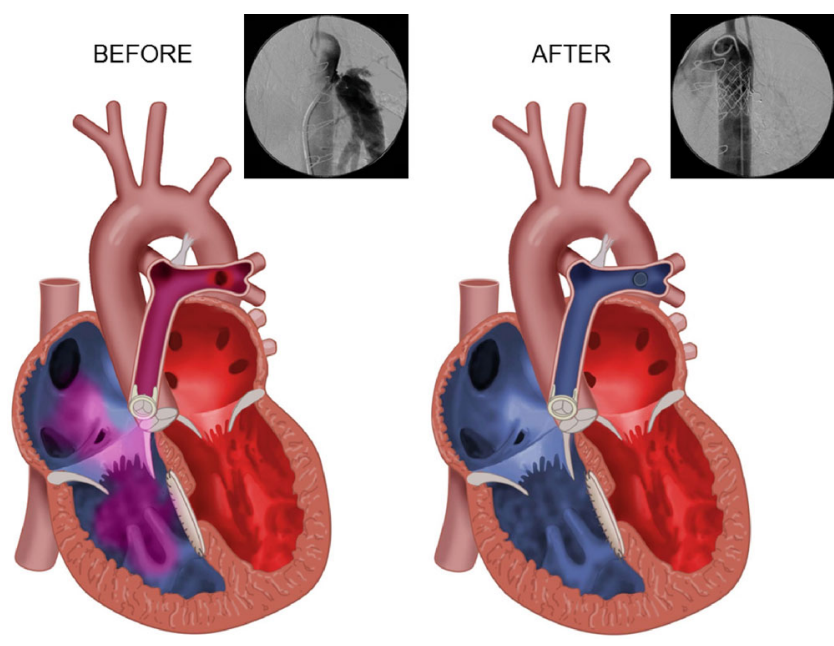

Fig. 1 Cardiac circulation of case 1 before and after closure of the Potts' shunt, including catheterisation images

However, eligibility for interventions strongly depends on the severity of the pulmonary hypertension and the ability of the pulmonary vasculature and right ventricle to adapt to resultant circulatory changes.

\section{Current consensus regarding invasive circulatory adjustments in adults with $\mathrm{PH}-\mathrm{CHD}$}

Publications regarding shunt adjustments in PH-CHD patients are scarce and mostly limited to rather general consensus guidelines [11, 12]. However, pre-interventional assessment of eligibility for intervention is mandatory, as shunt procedures contain a high risk of deteriorating RV overload in severe pulmonary hypertension. An irreversibly high PVR is generally considered a contraindication for shunt closure when a predominantly left-to-right shunt exists at rest $[2,11,13,14]$. This kind of shunt functions as a safety communication between the left and right heart through which systolic shunt reversal can occur during exercise, when systemic vascular resistance decreases in contrast to the fixed PVR. Closure of this type of shunt renders a decrease in RV afterload at rest. However, if the PVR remains high after closure, inability of shunt reversal during exercise can increase RV afterload and lead to progressive RV failure [5]. In general, a PVR below 6 Wood units (WU) is thought to be feasible for shunt intervention. If the PVR exceeds this value but is less than two-thirds of the systemic resistance, intervention can still be considered. In line with this, the effective left-right shunt ratio (Qp:Qs) should be at least 1.5 in the case of a bidirectional shunt, as this inversely relates to the ratio between systemic and pulmonary vascular resistance [2, 13-15]. RV dilatation, dysfunction and pulmonary regurgitation are other indications for intervention, although manifest RV failure inherently increases procedural risk [13]. Furthermore, invasive cor- rections remain controversial after PVR reduction has been achieved using targeted therapy for pulmonary hypertension [16-19]. When pulmonary hypertension persists after repair, the haemodynamic situation is comparable with idiopathic pulmonary arterial hypertension and the prognosis is deemed poor [6].

Patients with PH-CHD exhibit great diversity and complexity in anatomic anomalies. The above-mentioned criteria illustrate that criteria to determine eligibility for intervention can be contradictory in individual cases. The current study states that refinement in individual disease characteristics, adjacent to relevant guidelines, is necessary to define appropriate treatment. To that purpose, we present four PH-CHD patients with worsening RV overload and an ambiguous indication for intervention. Shunt adjustments were performed by experts in the field after the local Grown-Ups with Congenital Heart Disease (GUCH) working group reached consensus regarding patient-tailored treatment.

\section{Methods and results}

\section{Case 1}

A woman, born with Fallot's tetralogy, initially received a Potts' shunt to augment pulmonary flow and stimulate pulmonary development. After childhood a total correction with closure of the Potts' shunt and subsequently pulmonary valve replacement was performed (Fig. 1). During follow-up her clinical condition slowly declined and magnetic resonance imaging showed RV dilatation with an increase in end-diastolic volume (EDV) from 132 to $169 \mathrm{ml}$ in 4 years (upper normal value: $157 \mathrm{ml}$ based on sex, age and body surface area) [20]. Contrast-enhanced computed tomography revealed recanalisation of the Potts' shunt. At catheterisation a pulmonary arterial pressure (PAP) of $120 / 40 \mathrm{mmHg}$ was found with a left ventricular end-diastolic pressure of $12 \mathrm{mmHg}$. The PVR was 11.2 WU in the presence of a $1.7 \mathrm{l} / \mathrm{min}$ left-right shunt. Systemic blood pressure was 190/85 mmHg. Coiling of the shunt was technically unfeasible. Therefore, at the age of 49 years, two covered stents were implanted in the aorta occluding the Potts' shunt. After the procedure the PAP decreased to $100 / 35 \mathrm{mmHg}$. Subsequently an endothelin receptor antagonist was started resulting in a further decrease in PAP to $80 / 25 \mathrm{mmHg}$ with a PVR of $5.5 \mathrm{WU}$. She improved clinically and RV function remained reasonable with a stable EDV of $165 \mathrm{ml}$. RV stroke volume increased from 61 to $89 \mathrm{ml}$, tricuspid regurgitation decreased from grade III to I and resting heart rate decreased from 91 to $68 \mathrm{bpm}$. Currently she remains clinically stable with more than ten years of follow-up. 


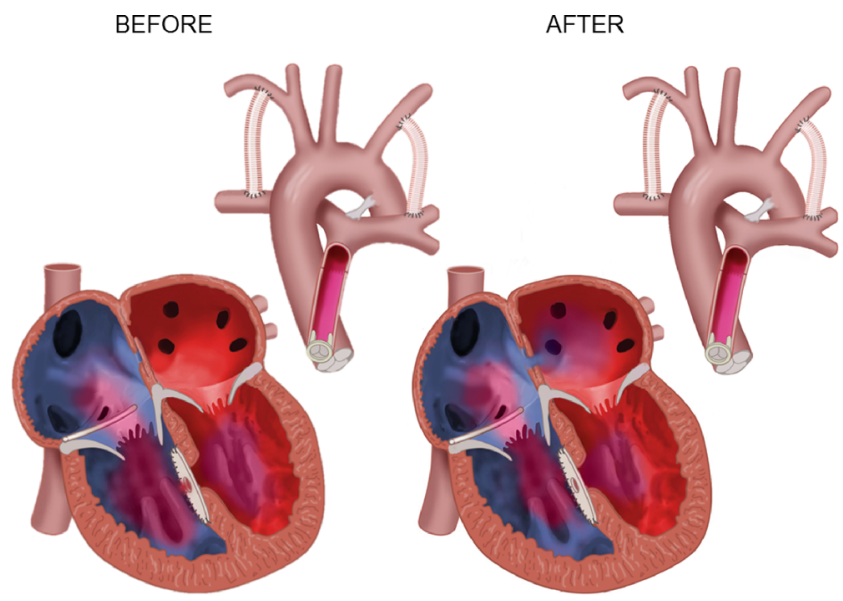

Fig. 2 Cardiac circulation of case 2 before and after balloon atrioseptostomy

\section{Case 2}

This woman was born with pulmonary atresia, a large VSD and major aortopulmonary collateral arteries (MAPCAs). She underwent consecutive placement of bilateral modified Blalock-Taussig shunts and ligation of MAPCAs. Subsequently a staged total correction, including fenestrated VSD closure, tricuspid valve plasty, RV outflow tract reconstruction and pulmonary valve replacement was performed. Pulmonary hypertension was diagnosed during follow-up and an endothelin receptor antagonist was administered. She developed progressive RV failure with multiple episodes of decompensation. Heart transplantation was denied because of thoracic deformities. RV overload deteriorated after a pulmonary infection, despite extensive diuretic and inotropic treatment. Echocardiography showed severe hepatic vein dilatation and a large quantity of ascites. She was 23 years old and her short-term prognosis was deemed to be very poor. It was decided to perform a balloon atrioseptostomy to relieve some RV overload by creating an additional shunt (Fig. 2). After intervention she gradually improved and the intravenous medication could be reduced. Systemic flow increased from 3.0 to 4.9 1/min. Furthermore, although systemic saturation dropped from 100 to $85 \%$, arterial oxygen supply increased from 453 to $629 \mathrm{ml} / \mathrm{min}$ through increased systemic flow. Unfortunately, four days after the procedure she developed a fatal ventricular rhythm disorder.

\section{Case 3}

A woman, born with Fallot's tetralogy, pulmonary atresia and a right-sided aortic arch, received bilateral BlalockTaussig shunts with ligation of MAPCAs. Subsequently the
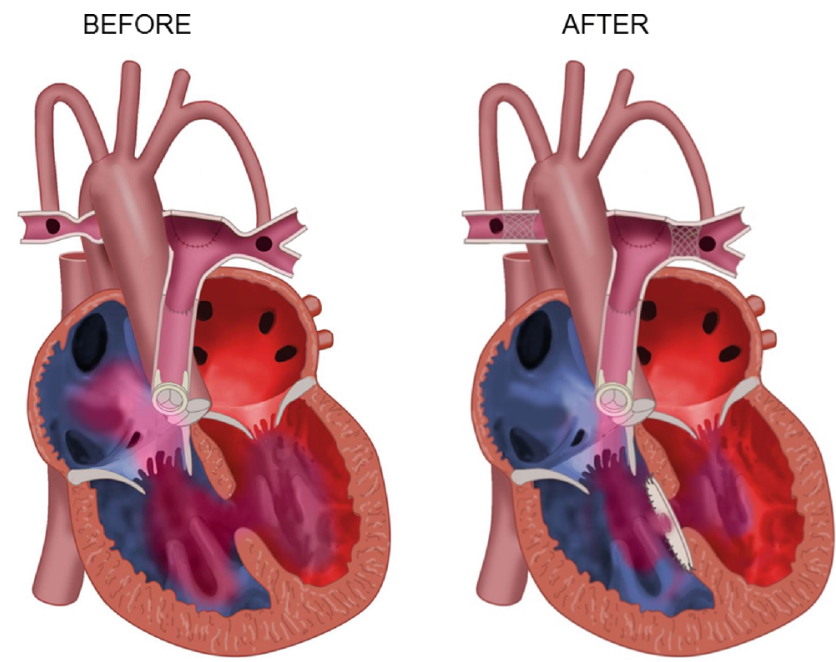

Fig. 3 Cardiac circulation of case 3 before and after pulmonary artery stenting and fenestrated VSD closure

RV outflow tract and pulmonary artery were reconstructed. During follow-up she developed progressive pulmonary hypertension resulting in a ventricular pressure equilibrium. PAP was $110 / 15 \mathrm{mmHg}$ in the presence of hypoplastic pulmonary vasculature and in combination with severe pulmonary regurgitation. The result was a predominantly right-to-left VSD shunt and Eisenmenger physiology with a haemoglobin level of $13.3 \mathrm{mmol} / \mathrm{l}$. Stenoses in the left and right pulmonary artery protected most of the peripheral pulmonary vasculature from pressure overload. At the age of 26 years the pulmonary vascular tree was corrected in a hybrid surgical and percutaneous procedure through pulmonary valve replacement and stenting of peripheral pulmonary stenoses. In the same session the VSD was reduced in size using a fenestrated patch (Fig. 3). After the operation the PAP decreased to $85 / 40 \mathrm{mmHg}$. Through pulmonary hypertension targeted therapy, the PAP dropped further to $75 / 20 \mathrm{mmHg}$. The Qp:Qs rate increased from 0.60 to 1.12 , haemoglobin level normalised to $9.5 \mathrm{mmol} / \mathrm{l}$ and peripheral arterial saturation increased from 82 to $90 \%$, all indicating a decline in de-oxygenated right-left shunt. After 7 years of follow-up her clinical condition currently remains stable.

\section{Case 4}

This woman was diagnosed with a double inlet left ventricle, a rudimentary right ventricle, ventriculo-arterial discordance, a large VSD, patent ductus arteriosus and pulmonary stenosis. A Blalock-Taussig shunt was placed at adult age. During follow-up she developed progressive dyspnoea with exercise-induced peripheral desaturation and became dependent on supplementary oxygen. Catheterisation showed a PAP of $87 / 43 \mathrm{mmHg}$ measured after the pul- 


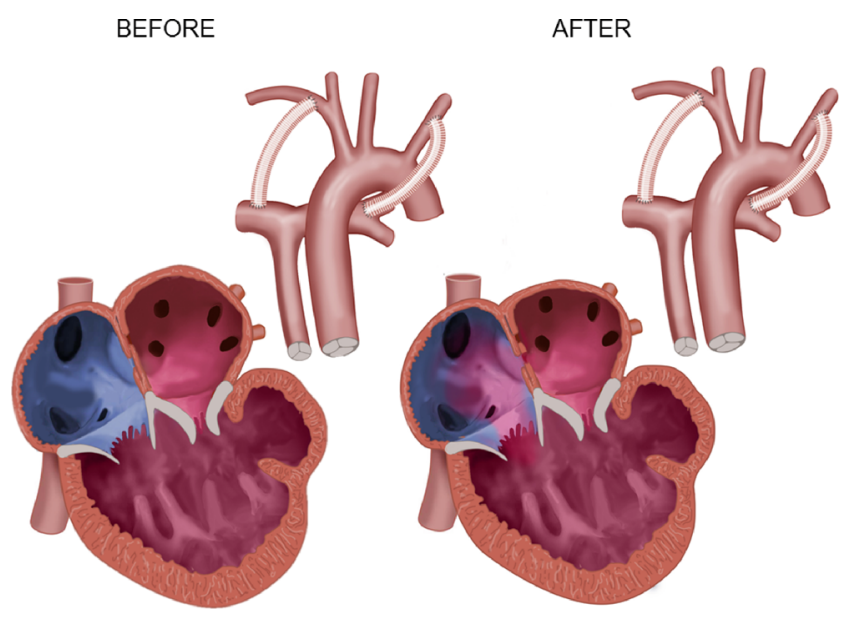

Fig. 4 Cardiac circulation of case 4 before and after graded balloon atrioseptostomy

monary stenosis with a PVR of 6.9 WU. Mean left atrial pressure was $20 \mathrm{mmHg}$ in the presence of a significant pulmonary venous atrioventricular valve stenosis. It was hypothesised that the elevated pulmonary venous pressure formed an important cause of her poor functional status. Balloon dilatation of the atrioventricular valve was deemed unfeasible, predominantly because of insufficient annulus support. Therefore, at the age of 47 years, a graded balloon atrioseptostomy was performed to create a restricted leftright atrial shunt that would relieve pulmonary congestion, improve pulmonary flow and thereby increase systemic saturation (Fig. 4). Since the monoventricle distributed flow over the pulmonary and systemic circulation inversely to their respective afterloads, the atrioseptostomy was made restrictive to prevent an excessive shunting volume and to protect the monoventricle and pulmonary circulation from flow overload. Left atrial pressure decreased from 20 to $14 \mathrm{mmHg}$ after the procedure, while right atrial pressure increased from 4 to $8 \mathrm{mmHg}$ and systemic saturation increased from 83 to $87 \%$. Pulmonary flow increased from 5.4 to $7.6 \mathrm{l} / \mathrm{min}$ and effective pulmonary flow (true flow of deoxygenated blood) increased from 2.2 to $3.0 \mathrm{l} / \mathrm{min}$. The remaining pulmonary hypertension was treated with an endothelin receptor antagonist. After intervention, unchanged diuretic treatment resulted in re-compensation and clinical improvement with an increase in six-minute walking distance from 179 metres before intervention to 336 metres 3 months post procedure. The created atrial septal defect turned out to decrease in size during follow-up and therefore the procedure was repeated twice more. Unfortunately, two years after the first atrioseptostomy she developed severe progressive heart failure with serious comorbidities and died after a hip fracture.

\section{Discussion}

This study presents four patients with complex CHD-PH, impaired pulmonary flow and progressive ventricular overload. All these patients had an ambiguous indication for shunt adjustment according to the current guidelines. In the first patient with corrected Fallot's tetralogy and recanalisation of a Potts' shunt, RV dilatation formed a class IB indication for intervention. On the contrary, the high PVR formed a strong contraindication and the remaining pulmonary hypertension would be associated with poor outcome $[2,13]$. However, in this patient the combined therapy of shunt closure and targeted therapy for pulmonary hypertension resulted in enhancement of RV function and long-term clinical improvement. The second patient with chronic right heart overload underwent an atrioseptostomy to increase systemic cardiac output. Guidelines do not comment on atrioseptostomy in the case of RV overload in PHCHD. However, this procedure is described in other types of pulmonary arterial hypertension with acceptable results [21, 22]. The procedure resulted in enhanced systemic oxygen delivery and short-term clinical improvement. However, it cannot be excluded that the decreased peripheral saturation contributed to the fatal arrhythmia. In the third patient with surgically palliated extreme Fallot's tetralogy, the predominantly right-left shunt and severe pulmonary hypertension formed strong contraindications for VSD closure [2, 13]. Fenestrated VSD closure reduced shunt volume, while maintaining a safety communication between the two ventricles [23]. In combination with pulmonary artery stenting and medical therapy a reduction in PAP and increase in pulmonary flow was achieved, resulting in enhanced systemic oxygen supply, normalisation of haemoglobin level and long-term clinical improvement. The last patient had a functional monoventricle with severe stenosis of the pulmonary venous atrioventricular valve for which a graded atrioseptostomy was performed. The literature states that intervention is indicated when symptomatic pulmonary venous obstruction is present [2]. However, the delicate equilibrium in this monoventricular circulation with combined arterial and venous pulmonary hypertension formed a high risk of cardiac deterioration if pulmonary overflow developed after intervention. Creation of a restrictive atrial shunt prevented an excessive increase in flow to the monoventricle and pulmonary circulation and resulted in improvement in pulmonary flow and clinical status.

In all cases the guidelines were inconclusive regarding eligibility for intervention. However, all these patients experienced progressive ventricular overload with diminished prognosis if left untreated. The local GUCH team reached consensus regarding a tailored invasive treatment strategy based on expert opinion adjacent to relevant guidelines. In these patients, intervention improved pulmonary flow and 
KEY MESSAGE

Invasive interventions in adults with congenital heart disease and pulmonary hypertension aim to optimize pulmonary flow and prevent right ventricular failure. This picture illustrates the wide variety in cardiac anomalies as well as the different mechanisms that can impair pulmonary flow. Current guidelines regarding interventions in this population are not extended enough to cover the wide pathophysiological spectrum. Therefore, patient tailored evaluation of disease characteristics is necessary adjacent to relevant guidelines to determine appropriate treatment. Prospective documentation and publications are warranted to further enhance insight in outcomes after intervention.

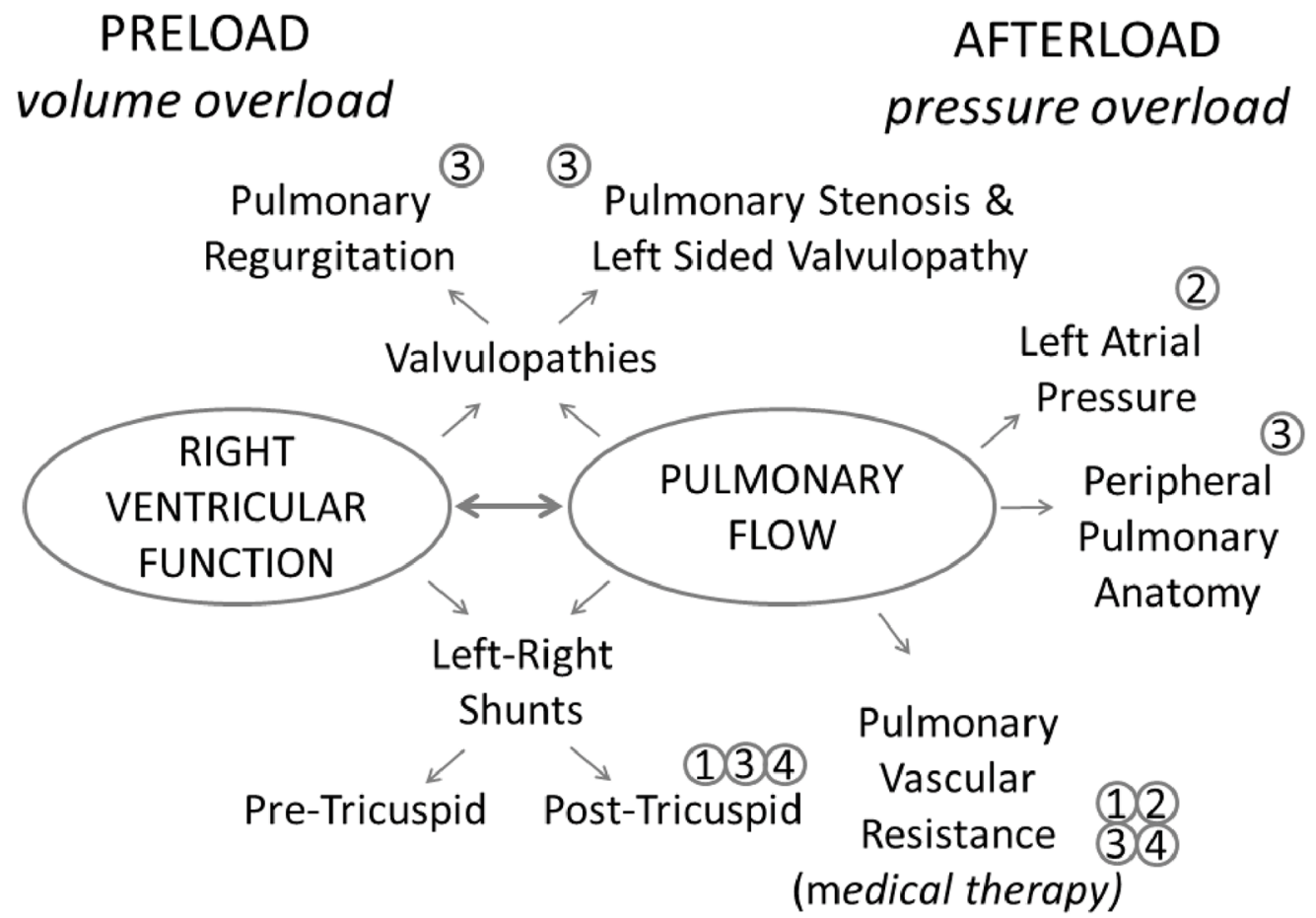

Fig. 5 Diversity in possible targets for invasive intervention in PH-CHD patients to optimise pulmonary flow and prevent RV failure, with the presented patients depicted by case numbers

clinical functioning. Currently, the guidelines are not extended enough to cover the wide pathophysiological spectrum of PH-CHD in the assessment of eligibility for intervention. Fig. 5 gives an overview of the diversity in possible targets for intervention in PH-CHD patients to optimise pulmonary flow and prevent RV failure, with the presented patients depicted by case numbers. This overview illustrates the wide variety in cardiac anomalies in this population as well as the different mechanisms that impaired pulmonary flow and were taken into account to determine treatment strategy in the presented cases. The current rise in adult patients with complex CHD-PH makes the debate about intervention increasingly relevant. In the past, similar case reports have been published concerning invasive interventions in patients with pulmonary hypertension and various forms of $\mathrm{CHD}$, which contribute to the statement that tailored interventions can improve clinical status when guidelines are inconclusive [24, 25]. More publications of individual experiences are warranted to further enhance insight into outcomes after intervention. Moreover, prospec- tive documentation of cases in preferably large registries is necessary to increase knowledge about the clinical course of this population and the effect of treatment strategies. Especially in the field of $\mathrm{GUCH}$, such registries have proven to be very useful for evaluation of outcome and best practice $[26,27]$. At present, we conclude that improvement in pulmonary flow and clinical functioning can be achieved in selected patients with complex CHD-PH through combination of tailored invasive and medical treatment.

Acknowledgment We wish to thank R. Slagter for the designs of Figures 1 to 4 .

Funding: The Department of Cardiology receives unrestricted grants from Actelion Pharmaceuticals Nederland BV (Woerden, the Netherlands), Biotronik (Berlin, Germany), Boston Scientific (Marlborough, Massachusetts) and Medtronic (Minneapolis, Minnesota).

Conflict of interest L.E. Couperus, I.R. Henkens, M.R.M. Jongbloed, M.G. Hazekamp, M.J. Schalij and H.W. Vliegen states that there are no conflicts of interest. 


\section{References}

1. Mulder BJ. Epidemiology of adult congenital heart disease: demographic variations worldwide. Neth Heart J. 2012;20:505-508.

2. Baumgartner H, Bonhoeffer P, De Groot NM, et al. ESC Guidelines for the management of grown-up congenital heart disease (new version 2010). Eur Heart J. 2010;31:2915-2957.

3. Marelli AJ, Mackie AS, Ionescu-Ittu R, Rahme E, Pilote L. Congenital heart disease in the general population: changing prevalence and age distribution. Circulation. 2007;115:163-172.

4. Mulder BJ. Changing demographics of pulmonary arterial hypertension in congenital heart disease. Eur Respir Rev. 2010;19:308313.

5. Huang JB, Liang J, Zhou LY. Eisenmenger syndrome: not always inoperable. Respir care. 2012;57:1488-1495.

6. Manes A, Palazzini M, Leci E, Bacchi Reggiani ML, Branzi A, Galie N. Current era survival of patients with pulmonary arterial hypertension associated with congenital heart disease: a comparison between clinical subgroups. Eur Heart J. 2014;35:716-724.

7. Duffels MG, Engelfriet PM, Berger RM, et al. Pulmonary arterial hypertension in congenital heart disease: an epidemiologic perspective from a Dutch registry. Int J Cardiol. 2007;120:198-204.

8. Gatzoulis MA, Beghetti M, Galie N, et al. Longer-term bosentan therapy improves functional capacity in Eisenmenger syndrome: results of the BREATHE-5 open-label extension study. Int J Cardiol. 2008;127:27-32.

9. Schulze-Neick I, Gilbert N, Ewert R, et al. Adult patients with congenital heart disease and pulmonary arterial hypertension: first open prospective multicenter study of bosentan therapy. Am Heart J. 2005;150:716.e7-716.e12.

10. Schuuring MJ, Boekholdt SM, Windhausen A, et al. Advanced therapy for pulmonary arterial hypertension due to congenital heart disease: a clinical perspective in a new therapeutic era. Neth Heart J. 2011;19:509-513.

11. Myers PO, Tissot C, Beghetti M. Assessment of operability of patients with pulmonary arterial hypertension associated with congenital heart disease. Circ J. 2014;78:4-11.

12. Gatzoulis MA, Alonso-Gonzalez R, Beghetti M. Pulmonary arterial hypertension in paediatric and adult patients with congenital heart disease. Eur Respir Rev. 2009;18:154-161.

13. Silversides CK, Dore A, Poirier N, et al. Canadian Cardiovascular Society 2009 Consensus Conference on the management of adults with congenital heart disease: shunt lesions. Can J Cardiol. 2010;26:e70-e79.

14. Lopes AA, O'Leary PW. Measurement, interpretation and use of haemodynamic parameters in pulmonary hypertension associated with congenital cardiac disease. Cardiol Young. 2009;19:431-435.

15. Kuijpers JM, Mulder BJ, Bouma BJ. Secundum atrial septal defect in adults: a practical review and recent developments. Neth Heart J. 2015;23:205-211.

16. Hirabayashi A, Miyaji K, Akagi T. Continuous epoprostenol therapy and septal defect closure in a patient with severe pulmonary hypertension. Catheter Cardiovasc Interv. 2009;73:688-691.

17. Ussia GP, Mule M, Caruso E, Aiello R, Tamburino C. Combined endothelin receptor antagonist and transcatheter interventional therapy of patent ductus arteriosus with severe pulmonary artery hypertension. Int J Cardiol. 2007; 116:427-429.

18. Mitropoulos FA, Apostolopoulou SC, Kanakis MA, Rammos S, Anagnostopoulos CE. Bosentan treatment in an adult with pulmonary hypertension due to patent ductus arteriosus permits surgical repair. J Heart Lung Transplant. 2007;26:1345-1346.

19. Frost AE, Quinones MA, Zoghbi WA, Noon GP. Reversal of pulmonary hypertension and subsequent repair of atrial septal defect after treatment with continuous intravenous epoprostenol. J Heart Lung Transplant. 2005;24:501-503.
20. Maceira AM, Prasad SK, Khan M, Pennell DJ. Reference right ventricular systolic and diastolic function normalized to age, gender and body surface area from steady-state free precession cardiovascular magnetic resonance. Eur Heart J. 2006;27:2879-2888.

21. Sandoval J, Gaspar J, Pena H, et al. Effect of atrial septostomy on the survival of patients with severe pulmonary arterial hypertension. Eur Respir J. 2011;38:1343-1348.

22. Allcock RJ, O'Sullivan JJ, Corris PA. Atrial septostomy for pulmonary arterial hypertension. Heart. 2003;89:1344-1347.

23. Novick WM, Sandoval N, Lazorhysynets VV, et al. Flap valve double patch closure of ventricular septal defects in children with increased pulmonary vascular resistance. Ann Thorac Surg. 2005;79:21-28.

24. Eicken A, Balling G, Gildein HP, Genz T, Kaemmerer H, Hess J. Transcatheter closure of a non-restrictive patent ductus arteriosus with an Amplatzer muscular ventricular septal defect occluder. Int J Cardiol. 2007;117:e40-e42.

25. Yamauchi H, Yamaki S, Fujii M, Iwaki H, Tanaka S. Reduction in recalcitrant pulmonary hypertension after operation for atrial septal defect. Ann Thorac Surg. 2001;72:905-906.

26. Drenthen W, Boersma E, Balci A, et al. Predictors of pregnancy complications in women with congenital heart disease. Eur Heart J. 2010;31:2124-2132.

27. van der Velde ET, Vriend JW, Mannens MM, Uiterwaal CS, Brand $\mathrm{R}$, Mulder BJ. CONCOR, an initiative towards a national registry and DNA-bank of patients with congenital heart disease in the Netherlands: rationale, design, and first results. Eur J Epidemiol. 2005;20:549-557. 